\title{
It is Time to Include Telehealth in Our Measure of Patient Retention in HIV Care
}

\author{
Dima Dandachi ${ }^{1,2}$. Jennifer Freytag ${ }^{3,4,5} \cdot$ Thomas P. Giordano $^{4,5,6} \cdot$ Bich N. Dang ${ }^{4,5,6}$
}

Published online: 27 April 2020

๑) Springer Science+Business Media, LLC, part of Springer Nature 2020

The definition of retention in HIV care has changed over the years, and different measures have been used to evaluate patient engagement in medical care. No gold standard exists for measures of retention, and each measure has its own set of advantages and limitations; however, all of these measures center around in-person clinic visits, including measures of visit frequency, gaps between visits, and so on [1-3]. We are now facing a pandemic of SARS-CoV-2 that is disrupting our healthcare system and the way we deliver face-to-face care. The pandemic has forced us to undergo a drastic and rapid switch to telehealth for most of our patient visits. This reduces risk of exposure to SARS-CoV-2 in the clinic setting and with travel to and from clinic [4]. In this pandemic, telemedicine has emerged, out of necessity, as a tool for safely keeping our patients engaged in care. Its use challenges traditional measures of retention and offers a unique opportunity to study telehealth as a new tool for keeping patients in HIV care.

Dima Dandachi

dandachid@health.missouri.edu

1 Division of Infectious Diseases, Department of Medicine, University of Missouri, Columbia, MO, USA

2 Department of Management, Policy, and Community Health, The University of Texas School of Public Health, Houston, TX, USA

3 Section of Health Services Research, Department of Medicine, Baylor College of Medicine, Houston, USA

4 VA Center for Innovations in Quality, Effectiveness and Safety (IQuESt), Houston, TX, USA

5 Michael E. DeBakey Veterans Affairs Medical Center, Houston, TX, USA

6 Section of Infectious Diseases, Department of Medicine, Baylor College of Medicine, Houston, TX, USA

\section{Digital Transformation}

Our clinic in Missouri is the only HIV clinic for hundreds of miles; patients drive hours for HIV care. We have been interested in expanding telehealth services for years, but COVID-19 accelerated what would have taken many years to a matter of a few days. In the midst of the pandemic, our HIV clinic has converted virtually all scheduled clinic visits to telehealth visits. We use Zoom ${ }^{\circledR}$ for video visits with end-to-end encryption and password protection. If a video visit is not feasible, we switch to audio only. We use landline or mobile telephone to connect with a small number of patients (less than 10\%) who do not have smart phones. During the visits, providers review labs, make sure patients have enough HIV medicines, and offer home delivery of medicines. Physical exams are not possible since patients are not equipped with telemonitoring devices.

\section{Patient Telehealth Experiences}

In our full transition to telehealth over the past month, we have had the chance to assess patients' attitudes towards its use. Many patients feel relieved to avoid travel and virtually all are relieved to avoid physical contact with the clinic. Overall, patients have positively embraced telehealth. Following a telehealth visit, our providers ask patients whether they liked the telehealth visit and how they rank the telehealth visit when compared to in-clinic visits (e.g. better, just as good, or worse) [5]. More than $90 \%$ of patients liked telehealth and ranked it as better or just as good as traditional in-clinic visits. These results suggest that patients view telehealth visits positively, and initial implementation in our clinic has been successful.

Our data on patients' attitudes towards using telehealth align with our team's prior research on willingness to use. In 2018, we asked patients at an HIV clinic in Houston about their attitudes towards using telehealth for HIV care. We 
posited that telehealth could address common barriers to retention in HIV care, including difficulties with transportation, inflexible work schedules, stressful life events, and stigma and fear of being seen at the HIV clinic. Overall, patients had a positive attitude and reported many potential benefits including better fit with work/ life schedules, reduced travel time, and having more privacy with the visit at home. However, $23 \%$ of patients had concerns about not being able to express themselves well, $37 \%$ had concerns about not having a physical exam, and $28 \%$ had concerns about the safety of their personal information over the internet. Interestingly, older age, lower income, and uncontrolled HIV (i.e., CD4 < 200) had no impact on the patients' attitude towards telehealth. However, patients who do not speak English as a first language and those with less education were less willing to use telehealth [6].

\section{Provider Telehealth Experiences}

In past studies on telehealth, providers were concerned about not being able to do a physical exam, longer visit times, and inability to connect with the patient $[7,8]$. While switching to telehealth during the COVID-19 pandemic has required a steep mental shift, most of our providers have found telehealth an acceptable alternative and easy to use. The time allocated for visits has been sufficient, and there have been few if any technical problems. A delightful upside to videobased visits is the provider's ability to see their patient's home and the social context of their patient's environment. These include family members who patients invite to join the call, but who otherwise would not have attended a visit. Visits are often less formal, and patients seem more relaxed in the comfort of their homes, opening up more, smiling more. Additionally, patients who often forget to bring their medicine bottles or do not remember the names of the medicines they take can easily retrieve their medicines and confirm what they are taking. In general, providers felt they were able to communicate their message well with patients. However, most visits were conducted with established patients who the providers already know and with whom they have rapport.

\section{Telehealth and Patient Retention}

Telehealth has the potential to improve engagement in HIV care, particularly in hard-to-reach populations for whom transportation and stigma remain prominent barriers. For such patients, "showing up" to a digital visit may prove easier. In fact, during this COVID-19 pandemic, our clinic has had a higher rate of connecting with patients who are not usually retained in care. Telehealth may also prove a just as good or better medium for connecting with younger patients, who have grown up with smart phones and electronic applications.

\section{What Does the Future Look Like?}

Telehealth use has skyrocketed in the midst of this pandemic and will likely remain even after the pandemic ceases. While patients and providers tend to view telehealth favorably during this crisis, we must consider how this will look in the long term, post-pandemic. Many questions remain. Historically, healthcare systems have used telehealth as a tool to increase access for patients who have not had good access to HIV care (e.g., rural patients and prison populations). However, when rolled out to the general population of patients with HIV infection, will certain subpopulations benefit more or less? Our team has published data showing the importance of effective communication and patient experience in predicting retention in HIV care [9-11]. In using telehealth, we must consider how telehealth alters communication and patient experience, and how differences in communication and patient experience may impact long term adherence and retention. Even when patients and providers favor the use of telehealth, the structure and content of their conversations might be altered in ways that neither the patient nor provider recognize. If telehealth is here to stay, what will retention look like if both telehealth and in-person visits are used? The time to start thinking about telehealth in the context of retention is now.

Moving forward, we need to establish clear criteria for receipt of HIV care through telehealth. Patient-related factors include willingness to use telehealth and the ability to use digital communication technology. Disease related factors need to be studied. For example, should patients with new HIV diagnoses always be seen in clinic, regardless of HIV stage? Should we offer telehealth to patients with uncontrolled HIV? On one hand these patients are more likely to have serious disease, and historically, be scheduled more frequent clinic visits. However, these patients are also likely the ones who have the most barriers to attending clinic visits (e.g., transportation, life change events). Studies are needed to understand how telehealth use will impact clinical outcomes, and how it could be incorporated into existing performance measures of retention in HIV care. Understanding how telehealth impacts completion of preventive services such as vaccinations and cancer screening is also necessary.

In the rush of the pandemic, many providers embraced telehealth. However, many providers are drawn to HIV primary care for the long-term relationships and patientprovider aspects of medicine. In normal times, how does telehealth impact provider job satisfaction? 
Finally, will communication issues unique to telehealth affect long-term retention? Will the technological interface prevent the development of close relationships and trust? Previous studies have found that patients tend to feel rushed during telehealth visits [12], even though telehealth visits tend to be longer; providers use less encouraging language during telehealth visits [13]; and these visits tend to be provider-dominated [14]. If telehealth use continues post-pandemic, providers may need communication skills training specific to telehealth (e.g., acknowledging/validating emotions without the ability to use common non-verbal cues) $[9,10]$.

\section{Beyond Telehealth}

Telehealth in the midst of COVID-19 is part of a larger change in practice. In this pandemic, health systems have made huge paradigm shifts in how we deliver care to our patients with HIV infection. Many private insurance companies and government agencies, including the AIDS Drug Assistance Programs (ADAP) in Missouri and Texas, have automatically extended pharmacy refills for HIV medicines to every 60 or 90 days (instead of 30 days). Clinic pharmacies, such as ours, are now mailing patients their HIV medicines. Providers are checking safety labs and HIV viral loads less frequently. COVID-19 has forced health systems and providers to make these changes in an incredibly short period of time, and patients have relished them. The changes have also made providers rethink their practice. For example, given the high efficacy and excellent safety profile of the newer HIV medicines, do patients need safety labs and an HIV viral load every six months? Once the pandemic ends, health systems will also need to rethink their practice and ask if it makes sense to ever revert back. If we aren't turning back, how will health systems sustain these changes over time?

To end the HIV epidemic, we need to focus on finding new ways to improve HIV retention in care and treatment. The COVID-19 pandemic has accelerated the implementation of telehealth and many other systems-level practices. We should use the lessons learned from the field during this time and develop well-designed research to address unresolved questions and to improve patient care.

\section{References}

1. Mugavero MJ, Davila JA, Nevin CR, Giordano TP. From access to engagement: measuring retention in outpatient HIV clinical care. AIDS Patient Care STDS. 2010;24(10):607-13.
2. Understanding the HIV Care Continuum: CDC; July 2017. https:// www.cdc.gov/hiv/pdf/library/factsheets/cdc-hiv-care-continuum. pdf.

3. Reveles KR, Juday TR, Labreche MJ, Mortensen EM, Koeller JM, Seekins D, et al. Comparative value of four measures of retention in expert care in predicting clinical outcomes and health care utilization in HIV patients. PLoS ONE. 2015;10(3):e0120953.

4. Keesara S, Jonas A, Schulman K. Covid-19 and health care's digital revolution. N Engl J Med. 2020. https://doi.org/10.1056/ NEJMp2005835.

5. Polinski JM, Barker T, Gagliano N, Sussman A, Brennan TA, Shrank WH. Patients' satisfaction with and preference for telehealth visits. J Gen Intern Med. 2016;31(3):269-75.

6. Dandachi D, Dang BN, Lucari B, Teti M, Giordano TP. Exploring the Attitude of Patients with HIV About Using Telehealth for HIV Care. AIDS Patient Care STDS. 2020;34(4):166-72.

7. Anderson K, Francis T, Ibanez-Carrasco F, Globerman J. Physician's perceptions of telemedicine in HIV care provision: a cross-sectional web-based survey. JMIR Public Health Surveill. 2017;3(2):e31.

8. Dandachi D, Lee C, Morgan RO, Tavakoli-Tabasi S, Giordano TP, Rodriguez-Barradas MC. Integration of telehealth services in the healthcare system: with emphasis on the experience of patients living with HIV. J Investig Med. 2019;67(5):815-20.

9. Dang BN, Westbrook RA, Njue SM, Giordano TP. Building trust and rapport early in the new doctor-patient relationship: a longitudinal qualitative study. BMC Med Educ. 2017;17(1):32.

10. Dang BN, Westbrook RA, Black WC, Rodriguez-Barradas MC, Giordano TP. Examining the link between patient satisfaction and adherence to HIV care: a structural equation model. PLoS ONE. 2013;8(1):e54729.

11. Dang BN, Westbrook RA, Hartman CM, Giordano TP. Retaining HIV patients in care: the role of initial patient care experiences. AIDS Behav. 2016;20(10):2477-87.

12. Gordon HS, Solanki P, Bokhour BG, Gopal RK. I'm Not Feeling Like "I'm Part of the Conversation" patients' perspectives on communicating in clinical video telehealth visits. J Gen Intern Med. 2020. https://doi.org/10.1007/s11606-020-05673-w. [Epub ahead of print]

13. Liu X, Sawada Y, Takizawa T, Sato H, Sato M, Sakamoto H, et al. Doctor-patient communication: a comparison between telemedicine consultation and face-to-face consultation. Intern Med. 2007;46(5):227-32.

14. Street R, Wheeler EJ, McCaughan W. Specialist-primary care provider-patient communication in telemedical consultation. Telemed J. 2000;6(1):45-544.

Publisher's Note Springer Nature remains neutral with regard to jurisdictional claims in published maps and institutional affiliations. 